\title{
Influence of Capsaicinoids Content on the Microbial Community during Kimchi Fermentation
}

\author{
Boyeon Park ${ }^{\dagger}$, Ji-Su Yang ${ }^{\dagger}$, Eun Woo Moon, Hye-Young Seo, and Ji-Hyoung $\mathrm{Ha}^{*}$ \\ Hygienic Safety and Analysis Center, World Institute of Kimchi, Gwangju 61755, Republic of Korea
}

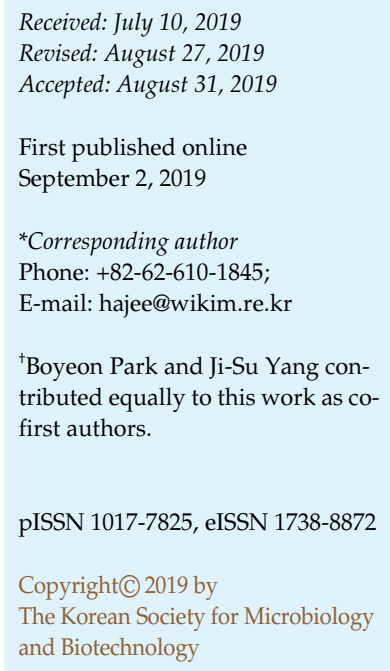

\begin{abstract}
Capsaicinoids in red pepper powder are known to show anti-bacterial effects; however, their effects during kimchi fermentation are not known. This study aimed to investigate the effects of various concentrations of capsaicinoids on kimchi fermentation. Five sets of kimchi samples were prepared using $0 \mathrm{mg} / \mathrm{kg}$ (control), $98.34 \pm 5.34 \mathrm{mg} / \mathrm{kg}$ (mild), $243.47 \pm 3.71 \mathrm{mg} / \mathrm{kg}$ (medium), $428.63 \pm 30.78 \mathrm{mg} / \mathrm{kg}$ (hot), and 1,320.49 $\pm 28.27 \mathrm{mg} / \mathrm{kg}$ (extreme) capsaicinoid. The characteristics of each kimchi sample, including $\mathrm{pH}$, acidity, organic acid, sugars, sugar alcohol, capsaicinoid content, and microbial community were periodically investigated during fermentation. Kimchi with red pepper powder shows significantly higher acidity than control kimchi, whereas $\mathrm{pH}$ values were the same. Organic acid in kimchi with red pepper powder was higher than in control kimchi, probably caused by higher lactic acid bacteria (LAB) counts in kimchi samples with red pepper powder. Our results show that addition of red pepper powder decreased Leuconostoc spp. counts in the bacterial community. In particular, Lactobacillus sakei and Leuconostoc gelidum counts increased and decreased, respectively, with increasing capsaicinoid content of red pepper powder added to kimchi. Overall, the results of this study indicate that physicochemical properties and LAB such as L. sakei and L. gelidum are influenced by capsaicinoid content. However, further studies are necessary to investigate the effects of the percentage of red pepper powder in kimchi on fermentation to provide practical guidelines for producing standardized kimchi.
\end{abstract}

Keywords: Capsaicinoids, kimchi, fermentation, microbial community

\section{Introduction}

Kimchi, listed in the Codex Alimentarius in 2001 (CODEX STAN 223-2001), is considered a vegetable probiotic food that promotes health benefits in a manner comparable to dairy probiotic foods such as cheese, kefir, drinking yoghurt, and bio-yoghurt [1]. Moreover, kimchi is most well-known as a minimally processed fresh food or non-thermally processed food because kimchi ingredients mainly include vegetables such as kimchi cabbage (Brassica rapa L. subsp. pekinensis), seasonings (red pepper, ginger, garlic, onion, and leek), and optional materials (various seafood or jeotgal), which cannot be pasteurized or sterilized during the kimchi-making process. Therefore, spontaneous fermentation results in the growth of miscellaneous lactic acid bacteria (LAB) during low- temperature storage leading to variety in advantageous sensory characteristics like kimchi flavor [2-7].

Various microorganisms involved in kimchi fermentation have been previously investigated. Previous studies on the taxonomic composition of bacterial communities from fresh or fermented kimchi have shown that several species of $\mathrm{LAB}$, including members of the genera Leuconostoc, Weissella, Lactococcus, Pediococcus, and Lactobacillus play important roles in determining the overall quality of kimchi products [8-11]. More recently, specific ingredients have been considered key factors in kimchi fermentation and could have a major influence during the fermentation process. Previous studies have reported the interrelationship between microbial succession and optional materials like jeotgal in kimchi during kimchi fermentation [6, 12-14]. In particular, 
microbial diversity in kimchi containing red pepper powder, which is a major kimchi ingredient, was analyzed using barcoded pyrosequencing analysis [6, 15]. However, the relationship between the microbial community and the level of red pepper powder spiciness during kimchi fermentation is unknown.

The latest food trends feature hot and spicy components in everything from main foods to desserts as well as in kimchi products. According to Kalsec's Annual Report \& Profile [15], the ratio of hot and spicy foods has increased by five percent points with a compound annual growth rate when comparing the years 2016 and 2017. There has been a twenty percent increase in the consumption of hot and spicy foods since 2007 across the globe. Overall, the consumers' preference and desire for spicier foods is increasing, along with interest in extremely hot kimchi. Interestingly, a previous study revealed that high content of capsaicinoids, an index of pungency, resulted in better salty taste perception compared to that with a low content of capsaicinoids in kimchi [16, 17]. These studies have resulted in much attention concerning the content of capsaicinoids, and many consumers consider that high content of capsaicinoids may contribute to reduce sodium intake. Several studies have verified that capsaicinoids affect the viability of microflora and even inactivate specific microorganisms [18-20]. Because the antimicrobial activity of capsaicinoids in red pepper powder is known [21, 22], capsaicinoids may be involved in microbial community and enrichment of taste and flavor. However, the influence of capsaicinoids during kimchi fermentation is not well understood. Therefore, it is important to precisely understand the role of capsaicinoid content in kimchi fermentation.

Here, we employed the Illumina MiSeq platform (Illumina, San Diego, CA, USA), which has been generally used for high-throughput sequencing, to monitor diverse microbial communities in kimchi. This study aimed to evaluate the influence of capsaicinoids at various concentrations on microbial communities and kimchi metabolites during kimchi fermentation. This study will provide a better understanding of the relation between capsaicinoid concentration and bacterial community profiles during kimchi fermentation.

\section{Material and Methods}

\section{Kimchi Preparation}

All the ingredients were purchased from the Gwangju agricultural products market and various red pepper powder products such as mild, medium, hot, and extreme depending on their capsaicinoid content were purchased from Dasan food (Korea). The kimchi used in the experiment was prepared according to the method of the World Institute of Kimchi with the following ratio: salted baechu $70 \%(\mathrm{w} / \mathrm{w})$; radish $8.82 \%(\mathrm{w} / \mathrm{w})$; green onion $3.18 \%(\mathrm{w} / \mathrm{w})$; red pepper powder $3.96 \%(\mathrm{w} / \mathrm{w})$; garlic $2.16 \%(\mathrm{w} / \mathrm{w})$; ginger $0.61 \%(\mathrm{w} / \mathrm{w})$; jeotgal $1.72 \%(\mathrm{w} / \mathrm{w})$; jindasi $0.9 \%(\mathrm{w} / \mathrm{w})$; glutinous rice $3.44 \%(\mathrm{w} / \mathrm{w})$; and water $5.21 \%$ $(w / w)$. Except for red pepper powder, the contents of all ingredients used in the manufacture of kimchi were the same amount. Five kimchi samples were stored at $6 \mathrm{C}$ for 6 weeks. Experiments were performed in triplicate (i.e., three independent kimchi containers were analyzed).

\section{Measurement of $\mathrm{pH}$ and Acidity}

Kimchi samples were homogenized using a blender (MQ775; Poland) and then filtered with sterilized gauze. The kimchi filtrate was used for $\mathrm{pH}$ and acidity measurements. The $\mathrm{pH}$ was determined using a pH meter (TitroLine 5000; SI Analytics GmbH, Germany) and acidity was titrated with $0.1 \mathrm{~N} \mathrm{NaOH}$ solution until the end point of $\mathrm{pH} 8.3$ was reached. Total acidity was calculated as a percentage of lactic acid.

\section{Determination of Capsaicinoid Contents}

For extraction of capsaicinoids, homogenized kimchi ( $2.5 \mathrm{~g})$ was weighed and added to glass beads (size 4) in $22 \mathrm{ml}$ clear vials equipped with PTFE liner (Supelco, USA) followed by $15 \mathrm{ml}$ of methanol. Vials were capped, placed in a heating block at $90^{\circ} \mathrm{C}$ for $1 \mathrm{~h}$, cooled at room temperature, transferred into $25 \mathrm{ml}$ volumetric flasks, and their volumes filled with methanol. Samples were filtered through disposable syringes with $0.2 \mu \mathrm{m}$ filters (Millipore, USA) and subjected to capsaicinoid content determination using HPLC with a Lachrom Ultra C18 column $(50 \mathrm{~mm} \times 2.0 \mathrm{~mm}, 2 \mathrm{um}$; Hitachi, Japan) and an Agilent 1260 infinity LC system equipped with a fluorescence detector (Agilent Technologies, USA). Analysis conditions were as follows: mobile phase A, 0.1\% acetic acid; mobile phase $\mathrm{B}$, acetonitrile; isocratic condition $\mathrm{A}: \mathrm{B}=6: 4$; flow rate of $0.6 \mathrm{ml} / \mathrm{min}$; excitation wavelength of $280 \mathrm{~nm}$, emission wavelength of $325 \mathrm{~nm}$; column temperature of $25^{\circ} \mathrm{C}$; and injection volume of $2 \mu \mathrm{l}$.

\section{Chemical Reagents}

For organic acids analysis, oxalic acid, citric acid, tartaric acid, malic acid, succinic acid, lactic acid, fumaric acid, and acetic acid (Sigma, USA) were used as standards. For sugars and sugar alcohol analysis, fructose (PHR1002-1 g), glucose (PHR1000-1 g), sucrose (47289-500 mg), maltose (PHR1497-1 g), mannitol (PHR1007-1 g), and sorbitol (PHR1006-1 g) standards were purchased from Sigma-Aldrich (USA). For capsaicinoid analysis, capsaicin (cat. 12084) and dihydrocapsaicin (cat. 03813) analytical standards were purchased from Sigma Aldrich. All solvents used for chromatographic analysis were HPLC grade and were purchased from J. T. Baker (USA). 


\section{Determination of Organic Acid Contents}

To measure the content of organic acids in kimchi, homogenized kimchi was filtered with a $0.2 \mu \mathrm{m}$ syringe filter (Milipore) and used to quantify organic acids. For HPLC analysis, Aminex $87 \mathrm{H}$ column $(300 \times 7.8 \mathrm{~mm}$, Bio-Rad, USA) and HPLCPDA (e2695-2998PDA, Waters, USA) were used. The analysis was performed in isocratic mode under the following conditions: mobile phase $0.008 \mathrm{~N} \mathrm{H}_{2} \mathrm{SO}_{4}$; flow rate of $0.6 \mathrm{ml} / \mathrm{min}$; column temperature of $50^{\circ} \mathrm{C}$; and injection volume of $10 \mu \mathrm{l}$.

\section{Measurement of $\mathrm{pH}$, Titratable Acidity, and LAB}

Kimchi samples were diluted ten-fold with $0.1 \%$ peptone water (BD Difco, USA) and inoculated in lactobacilli MRS agar (BD Difco) for $\mathrm{LAB}$ counting. $\mathrm{LAB}$ colonies were counted after each plate was sealed with a $\mathrm{CO}_{2}$ gas pack (GasPak Anaerobe Container System, USA) and incubated at $37^{\circ} \mathrm{C}$ for $48 \mathrm{~h}$.

\section{Determination of Sugars and Sugar Alcohol Contents}

Homogenized kimchi samples were squeezed using sterilized gauze, and sugars and sugar alcohols were extracted at $85^{\circ} \mathrm{C}$ for $25 \mathrm{~min}$ and cooled at room temperature. Extracted products were filtered with an $0.2 \mu \mathrm{m}$ syringe filter (Millipore) after centrifugation at 7,830 rpm for $10 \mathrm{~min}$ at $4^{\circ} \mathrm{C}$. For HPLC analysis, an Asahipak NH2P-50 4 E column (250 mm, $4.6 \mathrm{~mm}$, Shodex) and HPLC with a refractive index detector (RID) (Agilent Technologies, USA) were used. The analysis was performed in isocratic mode under the following conditions: mobile phase $75 \%$ $(\mathrm{v} / \mathrm{v})$ acetonitrile with water; flow rate of $1 \mathrm{ml} / \mathrm{min}$; column temperature of $30^{\circ} \mathrm{C}$; and injection volume of $6 \mu \mathrm{l}$.

\section{DNA extraction, DNA Amplification, and Illumina Sequencing}

Extraction of microbial DNA from kimchi samples was performed using a FastDNA SPIN kit.

The quality and concentration of extracted DNA were confirmed by $1 \%$ agarose gel electrophoresis and a NanoDrop UV spectrophotometer. Primers targeting the V3 to V4 regions of $16 \mathrm{~S}$ rRNA genes were used for PCR amplification with the extracted DNA. The primers used for microbial amplification were $341 \mathrm{~F}\left(5^{\prime}-\right.$ TCGTCGGCAGCGTC-AGATGTGTATAAGAGACAG-CCTACG GGNGGCWGCAG-3'; the underlined sequence indicates the target region primer) and 805R (5'-GTCTCGTGGGCTCGGAGATGTGTATAAGAGACAG-GACTACHVGGGTATCTAATCC$\left.3^{\prime}\right)$. Amplification conditions were as follows: initial denaturation at $95^{\circ} \mathrm{C}$ for $3 \mathrm{~min}$; followed by 25 cycles of denaturation at $95^{\circ} \mathrm{C}$ for $30 \mathrm{sec}$; primer annealing at $55^{\circ} \mathrm{C}$ for $30 \mathrm{sec}$; extension at $72^{\circ} \mathrm{C}$ for $30 \mathrm{sec}$; and a final elongation at $72^{\circ} \mathrm{C}$ for $5 \mathrm{~min}$. Secondary amplification for Illumina Miseq sequencing was then performed to attach the Illumina NexTera barcode to the PCR product with the i5 forward (5'-AATGATACGGCGACCACCGAGATCTACACXXXXXXXX-TCGTCGGCAGCGTC-3') and i7 reverse (5'-CAA GCAGAAGACGGCATACGAGAT- $X X X X X X X X$-GTCTCGTGGGCT CGG-3' ; X indicates the barcode region) primers. The conditions of the secondary amplification were the same as those of the previous one except that the number of amplification cycles was set to 8 . The PCR product purity was checked using $1 \%$ agarose gel electrophoresis and visualized using a Gel Doc system (BioRad).

The amplified PCR products were purified using CleanPCR (CleanNA, Netherlands). Purified products at equal concentrations were mixed together and non-target products were removed with CleanPCR (CleanNA, Netherlands). The size and quality of the product were checked on a Bioanalyzer 2100 (Agilent) using a DNA 7500 chip. Mixed amplicons were collected and sequenced using the Illumina MiSeq Sequencing system (Illumina, USA) at Chunlab, Inc. (Korea) according to the manufacturer's instructions.

\section{Processing of MiSeq Illumina Sequencing}

Processing of raw reads began with quality checking and filtering for low quality $(<\mathrm{Q} 25)$ using Trimomatic v0.32. After passing QC, paired-end sequence data were combined using PANDAseq. Primers were then trimmed with ChunLab's inhouse program at a similarity cut off of 0.8 . Amplicons contaning 16S rRNA sequences were identified using HMMER's hmmsearch program with the $16 \mathrm{~S}$ rRNA profiles. Sequences were denoised using DUDE-Seq and non-redundant reads were removed using UCLUST-clustering. The EzBioCloud database was used for taxonomic assignment using USEARCH (8.1.1861_i86linux32) followed by a more accurate pairwise alignment. To detect chimeras on reads containing less than $97 \%$ of the best hit similarity rate, UCHIME and the non-chimeric $16 \mathrm{~S}$ rRNA database from EzBioCloud were used. Sequence data were then clustered using CD-HIT8 and UCLUST.

\section{Results and Discussion}

\section{Capsaicinoid Content in Kimchi during Fermentation}

The capsaicinoid content in the samples is shown in Table 1. The mean values of capsaicin and dihydrocapsaicin contents from each product showed statistically significant differences $(p<0.05)$. The contents of capsaicin and dihydrocapsaicin, the major compounds determining the spicy taste of red pepper powder, were determined in five groups of kimchi: control, mild, medium, hot, and extreme kimchi. Capsaicin and dihydrocapsaicin were extracted with methanol and quantified using HPLC-FLD. Capsaicin and dihydrocapsaicin were qualitatively evaluated by comparing the retention time (RT) of the reference standard (Fig. 1). The retention time of capsaicin and dihydrocapsaicin was $2.5 \mathrm{~min}$ and $4.5 \mathrm{~min}$, respectively. The capsaicin and dihydrocapsaicin were not detected in the control kimchi (data not shown). In contrast, they were detected in kimchi containing red pepper powder. In mild 
Table 1. Capsaicin and dihydrocapsaicin content in red pepper powder for kimchi ingredients by HPLC-FLD.

\begin{tabular}{lccc}
\hline \multirow{2}{*}{ Product number } & \multicolumn{3}{c}{ Mean values $^{1)}(\mathrm{mg} / \mathrm{kg})$} \\
\cline { 2 - 4 } & Capsaicin & Dihydrocapsaicin & Capsaicinoids $^{2)}$ \\
\hline 1. Control & $\mathrm{ND}^{3)}$ & $\mathrm{ND}$ & $\mathrm{ND}$ \\
2. Mild level & $61.27 \pm 3.27^{\mathrm{a}}$ & $37.07 \pm 2.26^{\mathrm{a}}$ & $98.34 \pm 5.34^{\mathrm{a}}$ \\
3. Medium level & $143.83 \pm 2.25^{\mathrm{b}}$ & $99.63 \pm 1.49^{\mathrm{b}}$ & $243.47 \pm 3.71^{\mathrm{b}}$ \\
4. Hot level & $270.05 \pm 19.86^{\mathrm{c}}$ & $158.58 \pm 10.93^{\mathrm{c}}$ & $428.63 \pm 30.78^{\mathrm{c}}$ \\
5. Extreme level & $882.81 \pm 18.17^{\mathrm{d}}$ & $437.68 \pm 10.13^{\mathrm{d}}$ & $1320.49 \pm 28.27^{\mathrm{d}}$ \\
\hline
\end{tabular}

${ }^{1)}$ Values are mean \pm standard deviations of three $(n=3)$ measurements.

${ }^{2)}$ Capsaicinoids (capsaicin + dihydrocapsaicin).

${ }^{3)}$ Not detected.

${ }^{\mathrm{a}-\mathrm{d}}$ Different superscripts within a column indicate significant differences of the recovery rate, $p<0.05$.

kimchi, capsaicin and dihydrocapsaicin were in the range of $2.59-3.72 \mathrm{mg} / \mathrm{kg}$ and $1.46-2.14 \mathrm{mg} / \mathrm{kg}$, respectively and the average content of total capsaicinoids was $4.98 \pm$ $0.53 \mathrm{mg} / \mathrm{kg}$. In medium kimchi, capsaicin and dihydrocapsaicin were in the range of $5.30-6.82 \mathrm{mg} / \mathrm{kg}$ and $3.43-4.29 \mathrm{mg} / \mathrm{kg}$, respectively and the average content of total capsaicinoids was $9.98 \pm 0.77 \mathrm{mg} / \mathrm{kg}$. In hot kimchi, capsaicin and dihydrocapsaicin were in the range of 10.18$13.61 \mathrm{mg} / \mathrm{kg}$ and $5.35-7.19 \mathrm{mg} / \mathrm{kg}$, respectively. The average content of total capsaicinoids was $17.83 \pm 1.72 \mathrm{mg} / \mathrm{kg}$. In extreme kimchi, capsaicin and dihydrocapsaicin were in the range of $25.90-34.59 \mathrm{mg} / \mathrm{kg}$ and $12.32-16.43 \mathrm{mg} / \mathrm{kg}$, respectively, and the average content of total capsaicinoids was $43.68 \pm 5.82 \mathrm{mg} / \mathrm{kg}$. Total capsaicinoid content was high when the kimchi was made using red pepper powder with high-content capsaicinoids. No changes were observed in the content of capsaicinoids during kimchi fermentation. However, in extreme kimchi, difference in particles owing to the use of other seeds of red pepper powder are considered to be cause of deviation in the data. Our results suggest that the contents of capsaicinoids are directly related to the spicy taste of kimchi that contains red pepper powder. According to Dang et al. [23], capsaicin and dihydrocapsaicin are the main compounds that determine the spicy taste of red pepper powder.

\section{Changes in $\mathrm{pH}$ and Acidity of Fermented Kimchi}

Changes in $\mathrm{pH}$ and acidity during the fermentation of kimchi with different capsaicinoid content are shown in Fig. 2. The initial $\mathrm{pH}$ of the control kimchi without red

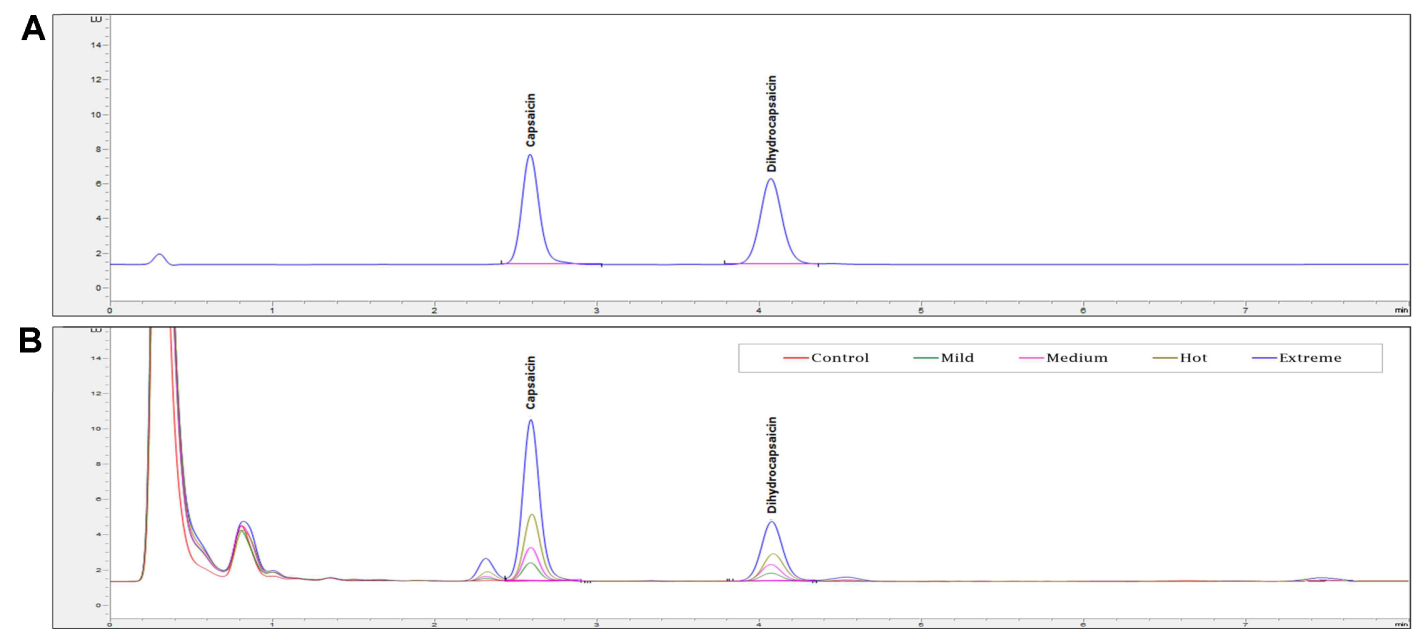

Fig. 1. HPLC chromatograms of: A, a standard solution containing capsaicin and dihydrocapsaicin; B, capsaicin and dihydrocapsaicin in five kinds of kimchi samples.

The LOD and LOQ for capsaicin were $0.18 \mathrm{mg} / \mathrm{kg}$ and $0.53 \mathrm{mg} / \mathrm{kg}$, respectively. The LOD and LOQ for dihydrocapsaicin were $0.11 \mathrm{mg} / \mathrm{kg}$ and $0.34 \mathrm{mg} / \mathrm{kg}$, respectively. 

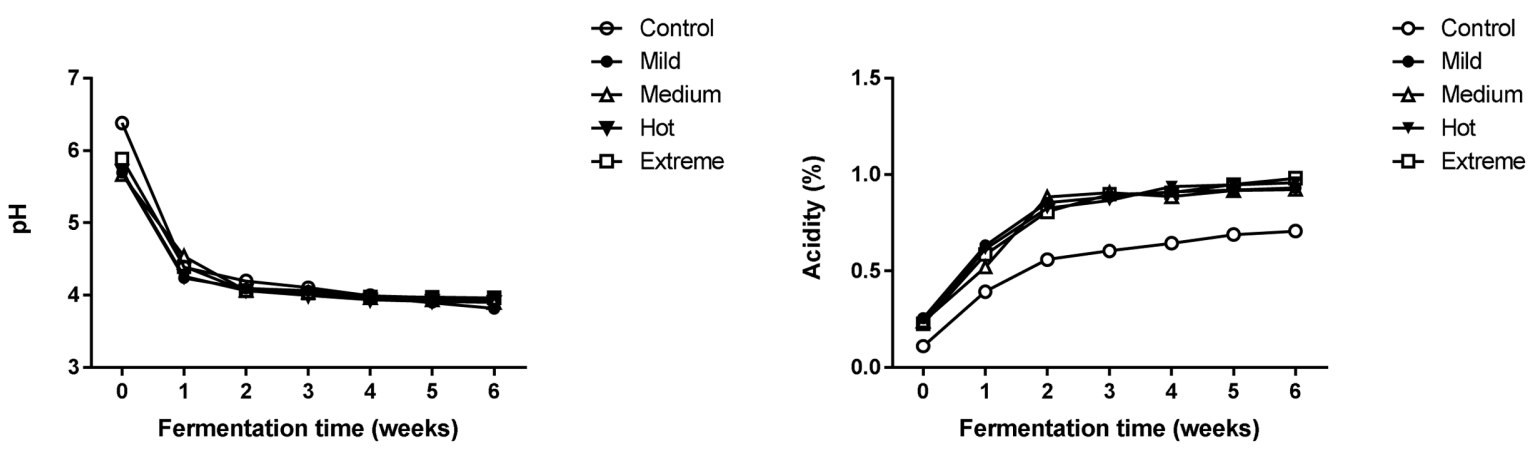

Fig. 2. Changes in $\mathrm{pH}$ and acidity of kimchi samples depending on various capsaicinoid content during 4 weeks of storage at $6^{\circ} \mathrm{C}$.

pepper powder was about 6.3 , which was higher than the initial $\mathrm{pH}$ of kimchi with red pepper powder (5.7-5.9). Our results indicate that the $\mathrm{pH}$ of kimchi is significantly affected by adding red pepper powder. Overall, the $\mathrm{pH}$ profiles of all kimchi samples decreased rapidly with ongoing lactic acid fermentation for 6 weeks, and the $\mathrm{pH}$ decreased slightly and became relatively stable in the range between 3.9 and 4.1 after 1 week. $\mathrm{pH}$ profiles of all kimchi samples were similar regardless of the addition of red pepper powder. Similarly, Jeong et al. [24] reported that the initial $\mathrm{pH}$ of kimchi with red pepper powder was lower than that of kimchi without red pepper power, and the $\mathrm{pH}$ value decreased rapidly during the initial kimchi fermentation period. They also demonstrated that red pepper powder plays a role in further lowering the initial $\mathrm{pH}$ value of kimchi, which is believed to delay kimchi fermentation at the initial stages. Our results show that the $\mathrm{pH}$ values of all kimchi samples with or without capsaicinoids were not remarkably different after 1 week.

The acidity of the control group increased from $0.1 \%$ to $0.7 \%$, whereas the kimchi added with red pepper powder presented higher acidity compared to control kimchi in the same time period, showing a range between $0.23 \%$ and $0.99 \%$. The $\mathrm{pH}$ and acidity of kimchi with red pepper powder over the 6 weeks fermentation period were similar to those in typical kimchi fermentation [15]. The acidities of the kimchi group treated with red pepper powder were significantly higher than the control throughout the fermentation period $(p<0.05)$. These results indicate that red pepper powder plays a role in changing the acidity value of kimchi during fermentation. Interestingly, the changes in acidity were significantly influenced by capsaicinoid levels $(p<0.05)$ (Fig. 2). The acidity of kimchi samples with red pepper powder was $0.90 \% \pm 0.00$ (mild), $0.93 \% \pm 0.00$ (medium), $0.96 \pm 0.01$ (hot), and $0.99 \% \pm 0.00$ (extreme hot) after 6 weeks of fermentation. According to several studies, kimchi has the most tempting taste, flavor, and texture when suitably fermented at between $0.6 \%$ and $0.7 \%$ titratable acidity or at a $\mathrm{pH}$ of approximately $4.4[25$, 26]. Notably, Park et al. [25] defined the $\mathrm{pH}$ of fresh kimchi, optimally fermented kimchi, and overfermented kimchi as $\mathrm{pH}$ 5.6, 4.4, and 3.7, respectively. In this study, the optimum fermentation time seems to be 1 or 2 weeks. Interestingly, the $\mathrm{pH}$ and acidity profiles were different although the pattern of profiles of $\mathrm{pH}$ and acidity should be similar in same kimchi samples (Fig. 2). According to Kim and In [27], however, the $\mathrm{pH}$ is not lowering below a certain limiting value even though lactic acid and acetic acid, which are mainly formed during kimchi fermentation, are accrued in kimchi samples because lactic acid and acetic acid are the weak acids. We assume that the different profiles of $\mathrm{pH}$ and acidity were caused by specific factors such as metabolites.

\section{Changes in Organic Acids Contents during Kimchi Fermentation}

The metabolites of kimchi such as organic acids and free sugars are influenced by the microbial community during the kimchi fermentation process and are important factors affecting the flavor and taste of kimchi $[24,28]$. The organic acid content in kimchi is shown in Fig. 3. The contents of lactic acid and acetic acid in all kimchi samples regardless of the addition of red pepper powder, ranged from $25.01 \pm$ 2.76 to $30.99 \pm 4.20 \mathrm{mg} / 100 \mathrm{~g}$ and from $27.04 \pm 1.00$ to 79.68 $\pm 3.13 \mathrm{mg} / 100 \mathrm{~g}$, respectively at the beginning of kimchi fermentation. Interestingly, the contents of acetic acid in control kimchi samples were lower compared to other kimchi samples. It is assumed that the acetate concentration difference between control kimchi and other kimchi samples are resulted from capsaicinoids in red pepper powder. The high acetic acid production of kimchi containing red pepper powder is thought to be due to 

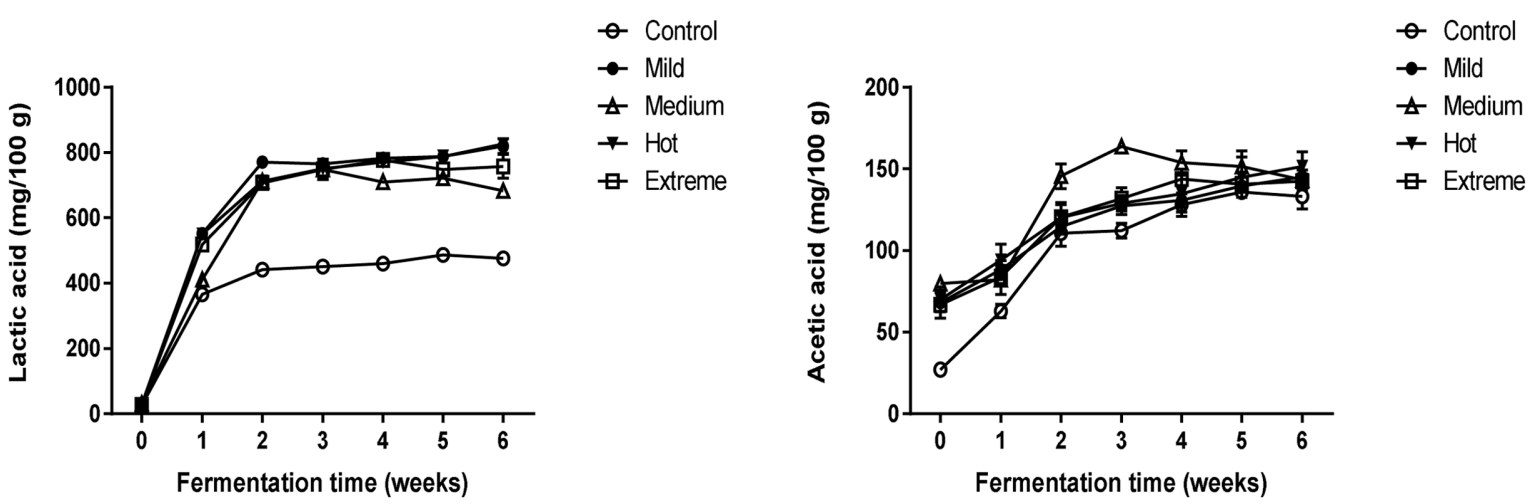

Fig. 3. Changes in organic acids content of kimchi samples depending on various capsaicinoid content during 6 weeks of storage at $6^{\circ} \mathrm{C}$.

microbial fermentation with high sugar and nutrients in the early stage of kimchi fermentation. Furthermore, no malic acid was detected after 1 week, even though all kimchi samples showed initial malic acid content in the range between $66.05 \pm 1.19$ and $129.85 \pm 2.41 \mathrm{mg} / 100 \mathrm{~g}$ (data not down). According to Yoo, Kim, \& Chung [29], malic acid is commonly converted to lactic acid and acetic acid by lactic acid bacteria during kimchi fermentation. Lactic acid and acetic acid levels increased sharply in the first 2 weeks and remained unchanged after 2 weeks. Interestingly, although no significant difference $(p>0.05)$ was observed in lactic acid content at the beginning of kimchi fermentation, lactic acid levels were lower in the control without red pepper than those in kimchi with red

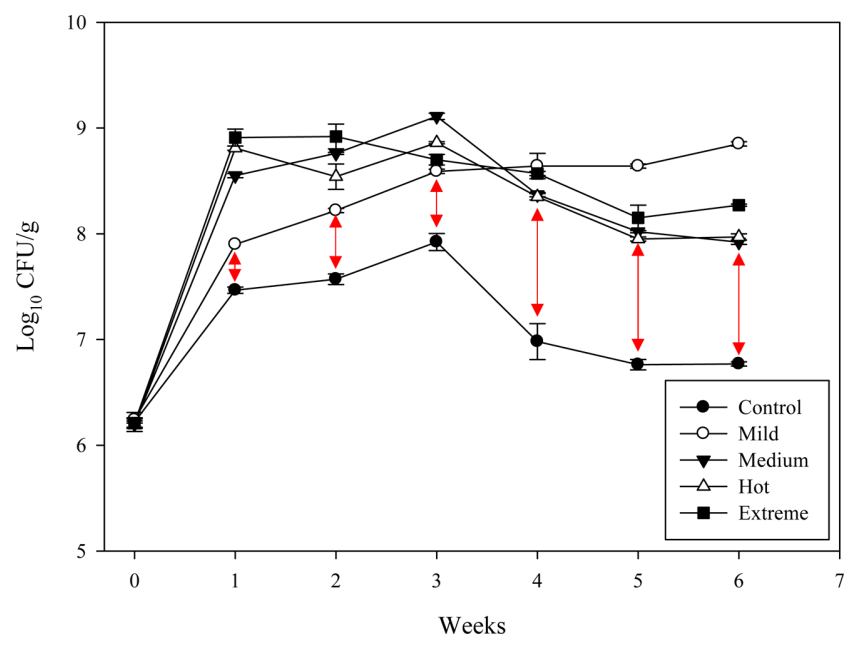

Fig. 4. Changes in lactic acid bacteria of kimchi depending on the concentration of capsaicinoids during 6 weeks of storage at $6^{\circ} \mathrm{C}$.

$(\mathbf{O})$ control; $(\bigcirc)$ mild; $(\boldsymbol{\nabla})$ medium; $(\triangle)$ hot; $(\boldsymbol{\square})$ extreme. pepper powder as fermentation time progressed to 6 weeks. However, no significant difference in lactic acid content was observed depending on the level of capsaicinoids in kimchi with red pepper powder $(p>0.05)$. In contrast, the level of acetic acid content exhibited significant differences at the beginning of kimchi fermentation, whereas no significant differences in acetic acid content was observed after 6 weeks $(p>0.05)$. Both lactic acid and acetic acid are major metabolites of heterofermentative $\mathrm{LAB}$ during vegetable fermentation, and the formation and accumulation of these organic acids decrease the $\mathrm{pH}$ and increase acidity during kimchi fermentation [25]. In this study, acidity and the contents of lactic acid and acetic acid showed similar trends. Notably, it is assumed that organic acids in kimchi samples with red pepper powder are higher than those in the control groups of kimchi without red pepper powder owing to the growth of LAB, which affected organic acid levels during fermentation. Fig. 4 shows the different levels of LAB between kimchi with and without red pepper powder. Previous studies reported that red pepper powder enhances the population of LAB in kimchi [30, 31]. Additionally, components of red pepper powder such as sugar can be used as a carbon source by LAB during kimchi fermentation. Moreover, in our result, mild kimchi showed the highest amount of total free sugar (Fig. 5). We assume that red pepper powder's contents affect LAB growth in kimchi with or without red pepper powder.

\section{Changes in Sugars and Sugar Alcohols during Kimchi Fermentation}

The sugar contained in kimchi is known to be a carbon source necessary for the growth of microorganisms. Free sugars are produced by the degradation of polysaccharide 
A

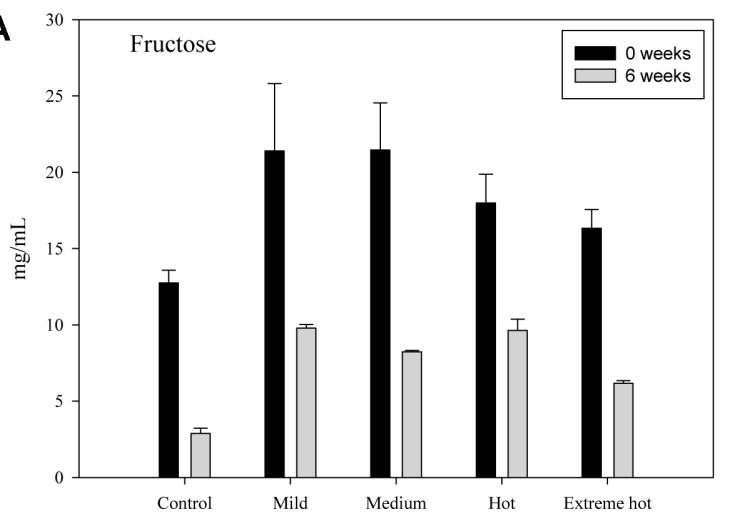

C

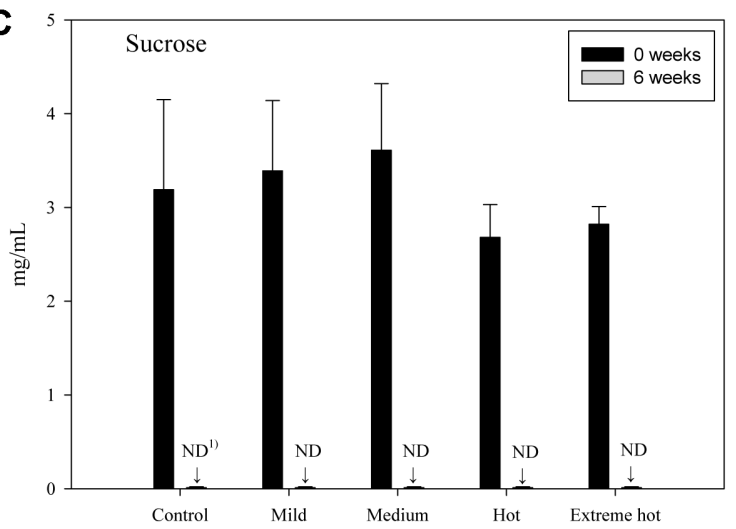

E

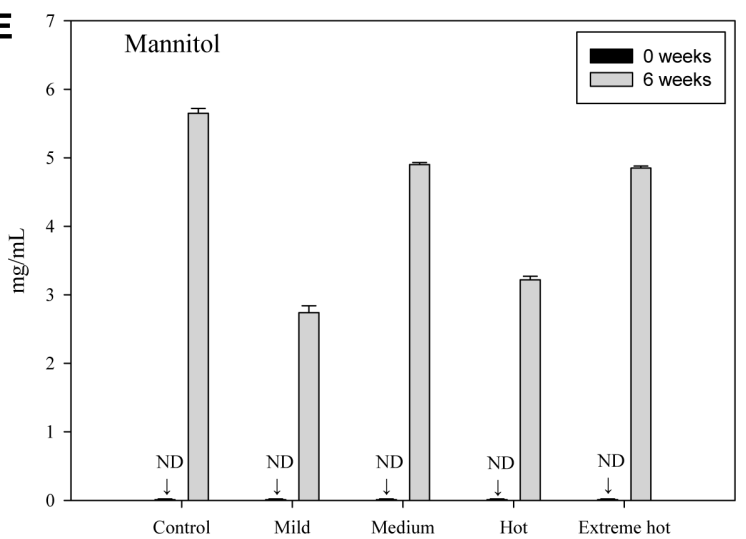

B

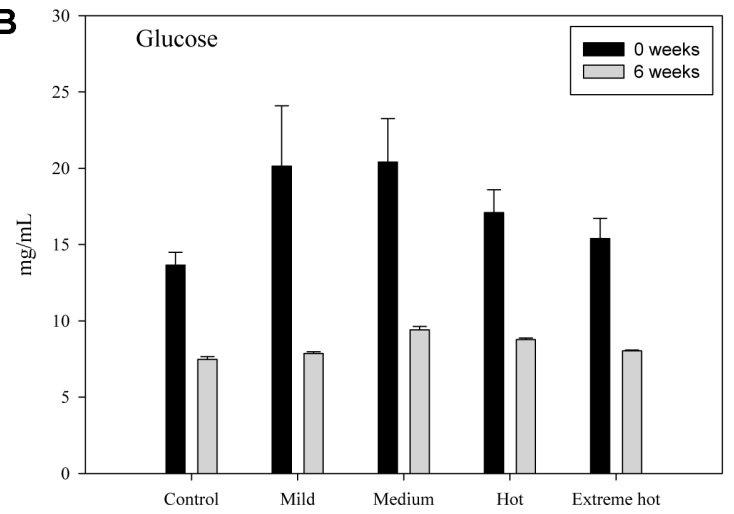

D

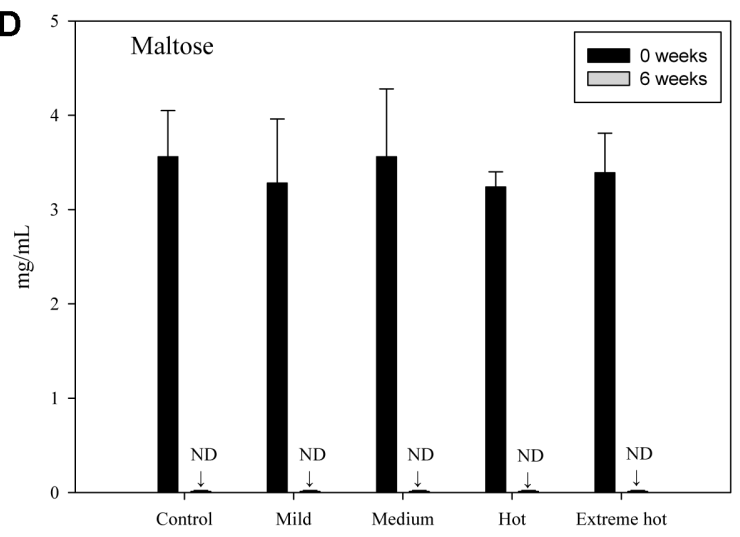

$\mathbf{F}$

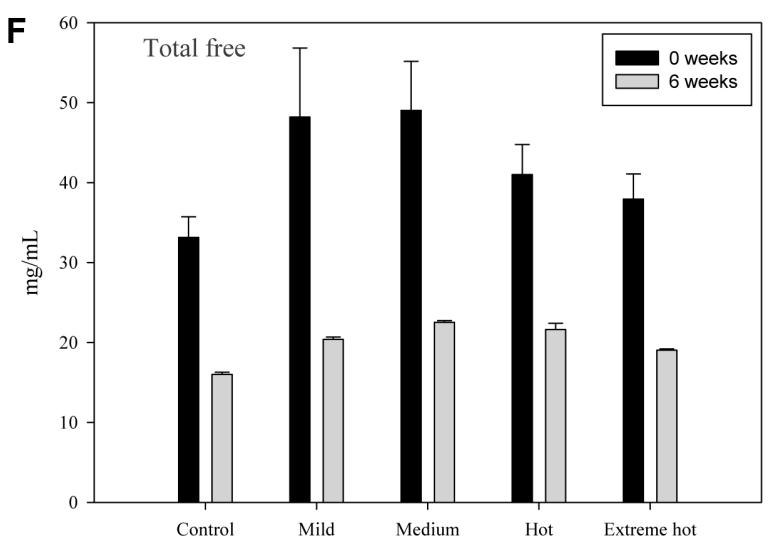

Fig. 5. Changes in free sugars of kimchi samples depending on various capsaicinoid content during 6 weeks of storage at $6^{\circ} \mathrm{C}$.

in cell wall of kimchi cabbage or originated from raw materials. Changes in free sugar content of kimchi with different capsaicinoid content during fermentation are shown in Fig. 5. The main free sugars were maltose, glucose, galactose, fructose, and mannitol; their sum is expressed as total sugar. Fructose and glucose accounted for the largest portion of free sugars in all kimchi samples and were detected at $13.7-20.4 \mathrm{mg} / \mathrm{ml}$ and $12.8-21.46 \mathrm{mg} /$ $\mathrm{ml}$, respectively, after manufacture (Fig. 5). Glucose and fructose were significantly lower in the control group than in the red pepper-contaning kimchi $(p<0.05)$, but there was no significant difference relative to capsaicinoid content as the fermentation progressed $(p>0.05)$. Mannitol, a sugar alcohol, was not initially detected, but increased after 2 weeks of fermentation, and showed significant levels in all kimchi samples with significant increases after 6 weeks $(p<0.05)$. In particular, the maximum level of mannitol was observed in the control group. Mannitol is 
reported to be produced from mannose and fructose by enzymatic action and fermentation [32]. In addition, Yun, Kang, and Song [33] reported that glucose is also liberated from kimchi cabbage and that most free sugars are transformed into mannitol as fermentation proceeds. In this study, with decreasing fructose and glucose levels during ongoing lactic acid fermentation, mannitol production in the kimchi samples seemed to be relatively increased. The total free sugar in all kimchi samples was $33.2-49.0 \mathrm{mg} / \mathrm{ml}$ at the beginning of fermentation. Kimchi containing red pepper powder showed higher total free sugar content compared to kimchi samples without red pepper powder, even though there was no significant difference relative to capsaicinoid content $(p>0.05)$. Free sugar content was markedly decreased at 1 week during fermentation, in agreement with the sharp change in $\mathrm{pH}$ and total acidity. During fermentation, there was no significant difference in the total free sugars depending on the concentration of capsaicinoids among the groups of kimchi with added red pepper powder $(p>0.05)$, and the total free sugar in control kimchi decreased to levels significantly lower than those in kimchi with red pepper powder. Microbial growth is generally active in the early stage of fermentation, when microorganisms rapidly consume sugar; the sugar content decreases gradually as fermentation progresses, leaving only a small amount. Therefore, the lower the sugar content, the more limited is the microbial activity and the lower the final acid production [34]. Overall, our results regarding exhaustion of total free sugar content imply that red pepper powder induces remarkable microbial activity and causes significant differences in physicochemical characteristics such as acidity, organic acids, sugars, and sugar alcohol in succession.

\section{Diversity of Microbial Community during Kimchi Fermentation}

To investigate the microbial community in five types of kimchi, MiSeq Illumina sequencing was performed. The average of sequencing reads with low-quality reads removed was 26,259 and the average length of reads was $414 \mathrm{bp}$. Based on the cutoff of $97 \%$ identity, the numbers of OTU in the samples ranged from 93 to 158 . The Good's coverage value, an estimate of sampling completeness for randomly selected amplicon sequences was $96.27-99.97 \%$, indicating that the number of sequencing reads obtained for each sample was sufficient to analyze microbial diversity. Alpha diversity values of the five types of kimchi were estimated by both the Shannon index (diversity) and Simpson and Chao1 index (richness) based on OTU profiles (Table 2). The results indicate that the diversity of the microbial community decreases during kimchi fermentation. The microbiological diversity showed a larger decrease in samples added with red pepper powder than in the control. This was confirmed by a decrease in the Shannon index and an increase in the Chao1 index in the corresponding samples.

Table 2. Summary of barcoded pyrosequencing datasets and statistical analysis of the five kimchi groups depending on capsaicinoid content.

\begin{tabular}{|c|c|c|c|c|c|c|c|c|}
\hline Sample & $\begin{array}{l}\text { Time } \\
\text { (weeks) }\end{array}$ & No. of reads & $\begin{array}{l}\text { Average read } \\
\text { length (bp) }\end{array}$ & OTUs & Shannon & Chao1 & Simpson & $\begin{array}{c}\text { Good's coverage of } \\
\text { library }(\%)\end{array}$ \\
\hline \multirow[t]{3}{*}{ Control } & 0 & 4,004 & 404.7 & 123 & 2.8 & 153.57 & 0.12 & 99.05 \\
\hline & 2 & 16,920 & 412.1 & 103 & 1.22 & 109.38 & 0.46 & 99.89 \\
\hline & 4 & 32,066 & 413.1 & 158 & 1.53 & 170.28 & 0.33 & 99.9 \\
\hline \multirow[t]{3}{*}{ Mild } & 0 & 2,020 & 404.4 & 146 & 3.57 & 193.04 & 0.05 & 97.57 \\
\hline & 2 & 30,926 & 418.3 & 98 & 0.71 & 101.5 & 0.7 & 99.95 \\
\hline & 4 & 64,742 & 423.1 & 151 & 0.77 & 158.39 & 0.62 & 99.96 \\
\hline \multirow[t]{3}{*}{ Medium } & 0 & 1,428 & 404.2 & 118 & 3.46 & 177.37 & 0.06 & 96.64 \\
\hline & 2 & 23,770 & 417.6 & 97 & 0.96 & 99.04 & 0.52 & 99.95 \\
\hline & 4 & 49,101 & 420.7 & 140 & 1.01 & 141.73 & 0.48 & 99.97 \\
\hline \multirow[t]{3}{*}{ Hot } & 0 & 1,127 & 404.1 & 126 & 3.76 & 173.83 & 0.04 & 96.27 \\
\hline & 2 & 20,813 & 417.5 & 93 & 0.81 & 96.9 & 0.63 & 99.94 \\
\hline & 4 & 60,996 & 422.6 & 147 & 0.8 & 154.16 & 0.58 & 99.96 \\
\hline \multirow[t]{3}{*}{ Extreme } & 0 & 1,348 & 404.1 & 156 & 4.06 & 189.18 & 0.03 & 96.44 \\
\hline & 2 & 27,772 & 417.5 & 95 & 0.78 & 98.64 & 0.62 & 99.95 \\
\hline & 4 & 56,846 & 421.8 & 116 & 0.7 & 122.42 & 0.62 & 99.96 \\
\hline
\end{tabular}




\section{Changes in Bacterial Composition of Kimchi Depending Capsaicinoid Contents}

To compare the changes in microbial community among samples, we confirmed the taxonomic structure at the genus and species levels (Fig. 6). At the genus level, the microbial community of the five types of kimchi showed
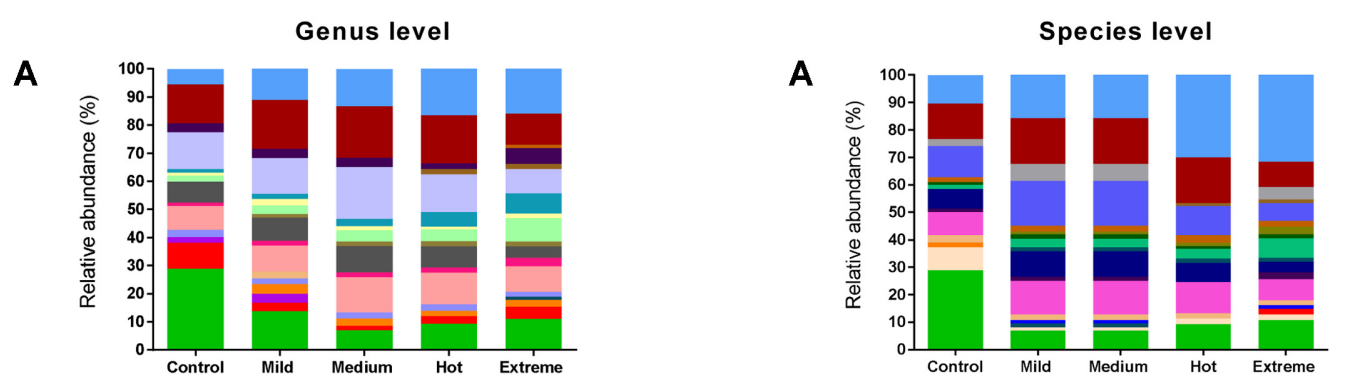

B

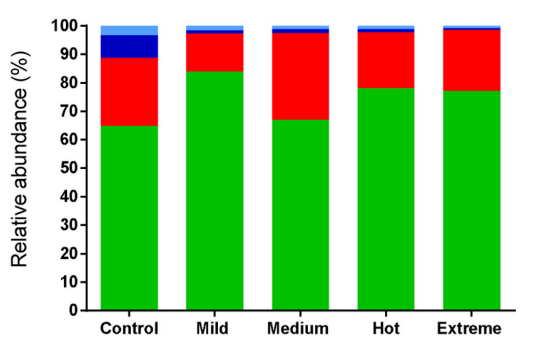

C
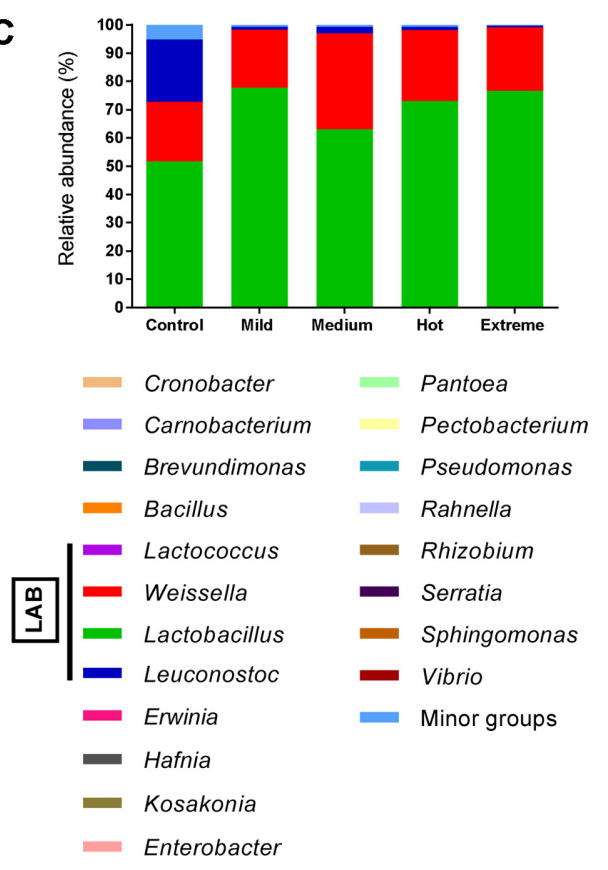

B

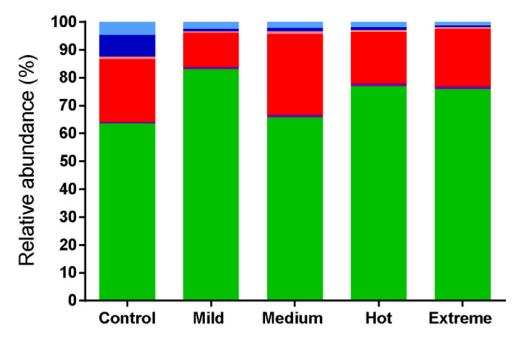

C

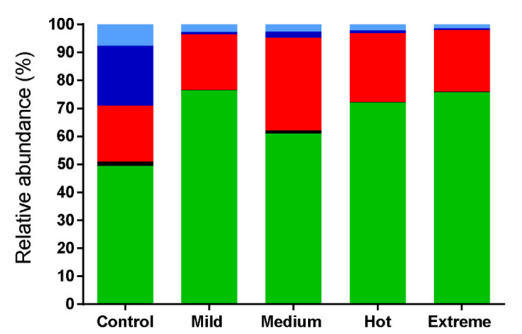

Rhizobium radiobacter group Enterobactergroup

- Rahnella woolbedingensis group Pseudomonas group

Rahnella aquatilis group Hafnia alvei group

- Pantoea agglomerans group Vibrio group

Kosakonia cowanii group Serratia group

- Pectobacterium carotovorum group Minor groups

- Erwinia persicina group

- Carnobacterium gallinarum group

- Bacillus subtilis group

- Bacillus licheniformis group

- Lactococcus lactis group

Weissella kandleri group

Weissella confusa group

Lactobacillus sakei group

¿ Lactobacillus_uc

- Lactobacillus algidus

- Leuconostoc gelidum group

Fig. 6. Bacterial taxonomic compositions showing the microbial successions of kimchi samples depending on various capsaicinoid content during 4 weeks of storage at $6^{\circ} \mathrm{C}$ at the genus and species levels.

Others are composed of taxonomic compositions showing less than $1 \%$ of the total reads in all respective samples in the genus and species level analyses. Samples of initial kimchi for 0 weeks, A; fermented kimchi for 2 weeks, B; fermented kimchi for 4 weeks, C. 
similar results in the early stage of fermentation, with Lactobacillus, Rahnella, and Enterobacter genera being predominant. As fermentation progressed, the dominance of Lactobacillus and Weisella accelerated. Interestingly, Leuconostoc genus during fermentation was dominant in the control without red pepper powder compared to those of kimchi samples with red pepper powder. At the species level, Lactobacillus sakei and Weissella kandleri predominated when kimchi with red pepper powder was fermented, and Leuconostoc gelidum was dominant in the control group compared to other samples. These results indicate that Lactobacillus, Weisella, and Leuconostoc may play a major role in kimchi fermentation. Jeong et al. confirmed the change in microbial community by adding red pepper powder; they found an increase in Weisella genus and a decrease in Leuconostoc and Lactobacillus genera [15]. Our results show that addition of red pepper powder resulted in a decrease in Leuconostoc in the bacterial community. Interestingly, Lactobacillus sakei increased and Leuconostoc gelidum decreased with increasing capsaicinoid content of red pepper powder added to kimchi. Based on these results, it may be considered that the ratio of Lactobacillus and Leuconostoc in the microbial community during kimchi fermentation may be changed by adding red pepper powder with regulated capsaicinoid-content during kimchi manufacture.

In this study, we analyzed the metabolites of kimchi and changes in microbial community composition of kimchi relative to capsaicinoid content in red pepper powder using a combination of biochemical characterization and MiSeq Illumina sequencing strategy to investigate the influence of capsaicinoid content in red pepper powder on kimchi fermentation. Our results clearly indicate that the groups with red pepper powder between mild to extremely hot levels for kimchi resulted in acceleration of LAB growth during the fermentation process. Moreover, lactic acid and acidity were significantly different between kimchi samples with and without red pepper powder. Lactobacillus sakei and Leuconostoc gelidum counts increased and decreased, respectively, with increasing capsaicinoid content of the red pepper powder added to kimchi. In particular, increasing the capsaicinoid content of red pepper powder in kimchi resulted in the maintenance of a lower abundance of Leuconostoc gelidum during fermentation. Further studies are needed to investigate the effects of the percentage of red pepper powder on kimchi fermentation, as well as the association between metabolites and bacterial communities and sensory characteristics such as kimchi flavors. Consequently, future plans will provide practical guidelines for producing standardized kimchi featuring both high quality and uniformity.

\section{Acknowledgments}

This research was supported by grants from the World Institute of Kimchi (KE1803-3 and KE1903-3), funded by the Ministry of Science and ICT, Republic of Korea.

\section{Conflict of Interest}

The authors have no financial conflicts of interest to declare.

\section{References}

1. Lee JH, Lee H, Kim SH, Eun JB, Ha JH. 2017. Recovery of intact human norovirus from cabbage Kimchi stored at $4^{\circ} \mathrm{C}$ and $10^{\circ} \mathrm{C}$ during fermentation. LWT Food Sci. Technol. 78: 258-264.

2. Kim M, Chun J. 2005. Bacterial community structure in kimchi, a Korean fermented vegetable food, as revealed by $16 \mathrm{~S}$ rRNA gene analysis. Int. J. Food Microbiol. 103: 91-96.

3. Cho J, Lee D, Yang C, Jeon J, Kim J, Han H. 2006. Microbial population dynamics of kimchi, a fermented cabbage product. FEMS Microbiol. Lett. 257: 262-267.

4. Chang HW, Kim KH, Nam YD, Roh SW, Kim MS, Jeon CO, et al. 2008. Analysis of yeast and archeal population dynamics in kimchi using denaturing gradient gel electrophoresis. Int. J. Food Microbiol. 126: 159-166.

5. Park JM, Shin JH, Lee DW, Song JC, Suh HJ, Chang UJ, et al. 2010. Identification of the lactic acid bacteria in kimchi according to initial and over-ripened fermentation using PCR and 16S rRNA gene sequence analysis. Food Sci. Biotechnol. 19: 541-546.

6. Jung JY, Lee SH, Kim JM, Park MS, Bae JW, Hahn Y, et al. 2011. Metagenomic analysis of kimchi, a traditional Korean fermented food. Appl. Environ. Microbiol. 77: 2264-2274.

7. Park EJ, Chun JS, Cha CJ, Park WS, Jeon CO, Bae JW. 2012. Bacterial community analysis during fermentation of ten representative kinds of kimchi with barcoded pyrosequencing. Food Microbiol. 30: 197-204.

8. Bae JW, Rhee SK, Park JR, Chung WH, Nam YD, Lee I, et al. 2005. Development and evaluation of genome-probing microarrays for monitoring lactic acid bacteria. Appl. Environ. Microbiol. 71: 8825-8835.

9. Kim J, Chun J, Han HU. 2000. Leuconostoc kimchii sp. nov., a new species from kimchi. Int. J. Syst. Evol. Microbiol. 50: 1915-1919. 
10. Lee JS, Heo GY, Lee JW, Oh YJ, Park JA, Park YH, et al. 2005. Analysis of kimchi microflora using denaturing gradient gel electrophoresis. Int. J. Food Microbiol. 102: 143-150.

11. Park EJ, Chang HW, Kim KH, Nam YD, Roh SW, Bae JW. 2009. Application of quantitative real-time PCR for enumeration of total bacterial, archaeal, and yeast populations in kimchi. J. Microbiol. 47: 682-685.

12. Jung MY, Kim TW, Lee C, Kim JY, Son HS, Kim YB, et al. 2018. Role of jeotgal, a Korean traditional fermented fish sauce, in microbial dynamics and metabolite profiles during kimchi fermentation. Food Chem. 265: 135-143.

13. Jung JY, Lee HJ, Chun BH, Jeon CO. 2016. Effects of temperature on bacterial communities and metabolites during fermentation of Myeolchi-Aekjeot, a traditional korean fermented anchovy sauce. PLoS One 11: e0151351.

14. Lee SH, Jung JY, Jeon CO. 2015. Bacterial community dynamics and metabolite changes in myeolchi-aekjeot, a Korean traditional fermented fish sauce, during fermentation. Int. J. Food Microbiol. 203: 15-22.

15. Jeong SH, Lee HJ, Jung JY, Lee SH, Seo HY, Park WS, J. et al. 2013. Effects of red pepper powder on microbial communities and metabolites during kimchi fermentation, Int. J. Food Microbiol. 160: 252-259.

16. Narukawa M, Sasaki S, Watanabe T. 2011. Effect of capsaicin on salt taste sensitivity in humans. Food Sci Technol. Res. 17: 167-170.

17. Chung Y, Jeong Y, Kim M, Lee Y. 2017. Effect of capsaicin on the threshold of sweet, sour, salty, and umami tastes. J. Korean Soc. Food Sci. Nutr. 46: 1510-1516.

18. Cowan MM. 1999. Plant products as antimicrobial agents. Clin. Microbiol. Rev. 12: 564-582.

19. Omolo MA, Wong ZZ, Mergen AK, Hastings JC, Le NC, Reiland HA, et al. 2014. Antimicrobial properties of chili peppers. J. Infect. Dis. Ther. 2: 1-8.

20. Zhou Y, Guan X, Zhu W, Liu Z, Wang X, Yu H, et al. 2014. Capsaicin inhibits Porphyromonas gingivalis growth, biofilm formation, gingivomucosal inflammatory cytokine secretion, and in vitro osteoclastogenesis. Eur. J. Clin. Microbiol. Infect. Dis. 33: 211-219.

21. Cichewicz RH, Thorpe PA. 1996. The antimicrobial properties of chile peppers (Capsicum species) and their uses in Mayan medicine. J. Ethnopharmacol. 52: 61-70.

22. Kusuda M, Inada K, Ogawa TO, Yoshida T, Shiota S, Tsuchiya T, et al. 2006. Polyphenolic constituent structures of Zanthoxylum piperitum fruit and the antibacterial effects of its polymeric procyanidin on methicillin-resistant Staphylococcus aureus. Biosci. Biotechnol. Biochem. 70: 1423-1431.

23. Dang YM, Hong YS Lee, CM. Khan N, Park S, Jeong SW, Kim KS. 2018. Determination of capsaicinoids in red pepper products from South Korea by high-performance liquid chromatography with fluorescence detection. Anal. Ytical Lett. 51: 1291-1303.

24. Jeong SH, Lee SH, Jung JY, Choi EJ, Jeon CO. 2013. Microbial succession and metabolite changes during longterm storage of Kimchi. J. Food Sci. 78: M763-769.

25. Park SK, Cho YS, Park JR, Moon JS, Lee YS. 1995. Changes in the contents of sugar, organic acid, free amino acid and nucleic acid-related compounds during fermentation of leaf mustard-Kimchi. J. Korean Soc. Food Sci. Nutr. 24: 48-53.

26. Lee YH, Yang IW. 1970. Studies on the packaging and preservation of kimchi. Appl. Biol. Chem. 13: 207-218.

27. Kim DC, In MJ. 2018. Quality characteristics of Mul-kimchi added with chlorella water extract. J. Appl. Biol. Chem. 61: 125-130.

28. Jung JY, Lee SH, Lee HJ, Seo HY, Park WS, Jeon CO. 2012. Effects of Leuconostoc mesenteroides starter cultures on microbial communities and metabolites during kimchi fermentation. Int. J. Food Microbiol. 153: 378-387.

29. Yoo MJ, Kim HR, Chung HJ. 2001. Changes in physicochemical and microbiological properties in low-temperature and long-term fermented kimchi during fermentation. J. Korean Soc. Food Cult. 16: 431-441.

30. Kang BK, Cho MS, Ahn TY, Lee ES, Park DS. 2015. The influence of red pepper powder on the density of Weissella koreensis during kimchi fermentation. Sci. Rep. 5: 15445.

31. Kang BK, Cho MS, Park DS. 2016. Red pepper powder is a crucial factor that influences the ontogeny of Weissella cibaria during kimchi fermentation. Sci. Rep. 6: 28232.

32. Ha JH, Hawer WS, Kim YJ, Nam YJ. 1989. Changes of free sugars in Kimchi during fermentation. Korean J. Food Sci. Technol. 21: 633-638.

33. Yun JW, Kang SC, Song SK. 1996. Mannitol accumulation during fermentation of kimchi. J. Biosci. Bioeng. 81: 279-280.

34. Park YH, Jung LH, Lee SS. 2001. Physicochemical characteristics of Toha-Jeot added cabbage Kimchi during fermentation. J. Korean Soc. Food Sci. Nutr. 30: 426-431. 